\title{
Perfil dos frequentadores e padrão de utilização das academias ao ar livre: revisão de escopo
}

\author{
Profile of users and usage pattern of outdoor gyms: scoping review
}

\section{AUTORES \\ Jéssica Aparecida Battistel ${ }^{1}$ (D) \\ Marilia Isabel Floss ${ }^{1}$ (D) \\ Agnes de Fátima Pereira Cruvinel $^{1}$ (D) \\ Paulo Roberto Barbato ${ }^{1}$ (D) \\ Rogério César Fermino 2,3 (D) \\ Paulo Henrique Guerra ${ }^{1}$ (D) \\ 1 Universidade Federal da Fronteira Sul, Chapecó, Santa Catarina, Brasil. \\ 2 Universidade Tecnológica Federal do Paraná, \\ Curitiba, Paraná, Brasil. \\ 3 Grupo de Pesquisa em Ambiente, Atividade Física e Saúde da Universidade Tecnológica Federal do \\ Paraná, Curitiba, Paraná, Brasil.}

\section{CONTATO}

Paulo Henrique Guerra

paulo.guerra@uffs.edu.br

Rodovia SC 484, Km 02, Chapecó, Santa

Catarina, Brasil.

CEP: 89815-899.

DOI

$10.12820 /$ rbafs. $26 \mathrm{e} 0186$

\section{(cc) BY}

Este trabalho está licenciado com uma Licença

Creative Commons - Atribuição 4.0 Internacional.

\begin{abstract}
RESUMO
Com objetivo de identificar e sumarizar os dados de pesquisas que investigaram o perfil dos usuários das Academias ao Ar Livre (AAL) no Brasil, assim como o padrão de utilização destes espaços, em dezembro de 2020 foi realizada uma revisão de escopo, procurando por artigos observacionais, conduzidos no território brasileiro, que identificaram o perfil dos frequentadores e o padrão de utilização das AAL. Dos 16 artigos encontrados, que representam 10 distintos projetos, identificou-se que mulheres, pessoas na faixa etária entre 40 e 59 anos de idade, com ensino médio completo, casados/as e com sobrepeso representam o perfil mais comum de frequentadores das AAL no país. Complementarmente, a síntese também aponta que frequentadores utilizam as AAL há, pelo menos, 12 meses, com periodicidade de utilização de, pelo menos, três dias da semana, geralmente, nos dias úteis. Em conclusão, as AAL contribuem para a equidade ao acesso à atividade física. Entretanto, novos projetos são necessários, com utilização de abordagens mistas, para uma melhor compreensão das características que ao momento ainda são inconclusivas.
\end{abstract}

Palavras-chave: Atividade física; Ambiente construído; Epidemiologia descritiva; Equidade em saúde; Revisão.

\begin{abstract}
In order to identify and summarize data from research which investigated the Profile of Outdoor Gyms (OG) users in Brazil, as well as the usage patterns of these spaces, in December 2020 a scoping review was developed, looking for observational studies, conducted in the Brazilian territory, which identified the users' profile and the usage pattern of $O G$. Of the 16 papers found, which represent 10 different studies, it was identified that women, people between 40 and 59 years of age, with complete secondary education, married and overweight represent the profile of the public who frequent $O G$ in the country. Complementarily, the synthesis also points out that frequenters have been using OG for at least 12 months, with periodicity of use of at least three days a week, usually on working days. In conclusion, the OG contribute to equity of access to physical activity. However, new studies are needed, using mixed approaches, to better understand the characteristics that are still inconclusive at the moment.
\end{abstract}

Keywords: Physical activity; Built environment; Epidemiology descriptive; Equity in healtb; Review.

\section{Introdução}

Pesquisas recentes apontam que a oferta de espaços públicos (ex. ruas de lazer, praças, parques e bosques) está associada à distintos indicadores positivos de saúde, nas diversas fases da vida ${ }^{1-3}$. Dentre estes, estão as Academias ao Ar Livre (AAL), que se caracterizam pela implementação de aparelhos e estruturas funcionais, em espaços públicos abertos, que permitem práticas estruturadas de atividade física (AF) de forma gratuita ${ }^{4}$. A gradual introdução das AAL no país emerge como uma estratégia do poder público em um momento em que há grande preocupação a respeito da equidade no acesso às $\mathrm{AF}$, uma vez que sua prática está relacionada ao desenvolvimento humano 5 .

No Brasil, mesmo com o aumento dos níveis re- comendados de AF moderadas e vigorosas nos últimos anos ${ }^{6,7}$, também se reconhece que a prática de $\mathrm{AF}$ está atrelada à uma série de fatores sociais, ambientais e econômicos ${ }^{6,8,9}$, o que, nesse sentido, torna profícua tanto a oferta, quanto a respectiva avaliação das ações e estratégias que tenham como objetivo a ampliação do seu acesso, de forma pública e gratuita.

Percebendo a disseminação das AAL no país, enquanto ação que visa a equidade de acesso à AF e melhoria dos indicadores de saúde, um dos passos iniciais de sua avaliação se dá pelo reconhecimento dos seus frequentadores, assim como do padrão de sua forma de utilização, entendendo-se que tal evidência pode contribuir com o processo de promoção da $\mathrm{AF}$ nestes espaços. Des- 
sa forma e, também em vista do seu debate no âmbito das políticas públicas ${ }^{10}$, buscou-se, por meio da presente pesquisa, levantar e sumarizar os dados de pesquisas que investigaram o perfil dos frequentadores das AAL no Brasil, assim como o padrão de utilização destes espaços.

\section{Métodos}

Foi conduzida uma revisão de escopo ${ }^{11}$, elaborada e escrita a partir das recomendações da lista PRIS$\mathrm{MA}^{12}$ e registrada na plataforma PROSPERO (CRD42019130189).

Como critérios de inclusão, foram procurados artigos científicos originais, de delineamento observacional, desenvolvidos no território brasileiro, que adotaram especificamente a terminologia "academia ao ar livre" e apresentaram o perfil dos seus frequentadores e o padrão de utilização das AAL (ex. sexo, faixa etária, renda, cor da pele, IMC, tempo de utilização e frequência de utilização das AAL, se pratica AF em outros locais), sem restrições quanto ao ano de publicação, faixa etária de interesse, níveis de AF e condição de saúde da população estudada, assim como em relação ao número de AAL estudadas, localizações, aparelhos e funcionamento.

Mesmo com o reconhecimento prévio de que as AAL são frequentadas, via de regra, por adultos e idosos, é importante mencionar que não foram considerados para a síntese os artigos científicos que utilizaram a terminologia "academia da terceira idade" (denominação das AAL em algumas cidades), pelo seu direcionamento específico e também em vista da possível influência em relação à faixa etária.

Os artigos em potencial foram procurados por três meios: (I) aplicação de buscas sistemáticas em sete bases de dados eletrônicas (Lilacs, Physical Education Index, Pubmed, Scielo, Scopus, Sportdiscus e Web of Science), no dia 15 de dezembro de 2020, partindo da estratégia desenvolvida para o Pubmed: ((((fitness zones[text word]) or fitness zone[text word]) or (public gym[text word])) or (outdoor gym[text word])) or (outdoor academ*[text word]). Nas bases Lilacs e Scielo, que indexam um grande número de periódicos científicos da América Latina e Caribe, as buscas também foram conduzidas com os termos em português; (II) leitura dos 150 primeiros resultados, ordenados por sua de relevância, no site Google Acadêmico (https://scholar.google.com.br/), partindo do termo "academia(s) ao ar livre" e (III) listas de referências dos artigos avaliados pelos seus textos integrais.

As estratégias de busca foram elaboradas de maneira independente por três pesquisadores (JB, MF e PG) e aplicadas nas bases de dados por um pesquisador (PG). As etapas de seleção dos artigos (por títulos, resumos e textos integrais) foram desenvolvidas por meio do trabalho independente de duas pesquisadoras (JB e MF), com auxílio de um terceiro pesquisador (PG), para estabelecer consensos. Pela sua característica descritiva, não foram projetados, desde os passos iniciais da presente revisão, a avaliação por risco de viés e a realização de metanálise.

O processo de extração dos dados originais, também conduzido por meio do trabalho independente de duas pesquisadoras (JB e MF), com auxílio de um terceiro pesquisador $(\mathrm{PG})$, foi realizado em uma planilha eletrônica, onde foram organizadas todas as informações descritivas relacionadas aos participantes das AAL. Ao término da extração de dados, se observou entre os artigos incluídos 86 distintas variáveis e, com vistas em apresentar um resultado mais robusto e objetivo, foi estipulado, mesmo que de maneira subjetiva, que as variáveis investigadas por, pelo menos, três projetos, seriam encaminhadas para a síntese descritiva. A planilha que detalha todas as variáveis identificadas e seus respectivos artigos/ projetos pode ser solicitada ao autor correspondente.

\section{Resultados}

Com a remoção das duplicatas, 1.035 artigos foram

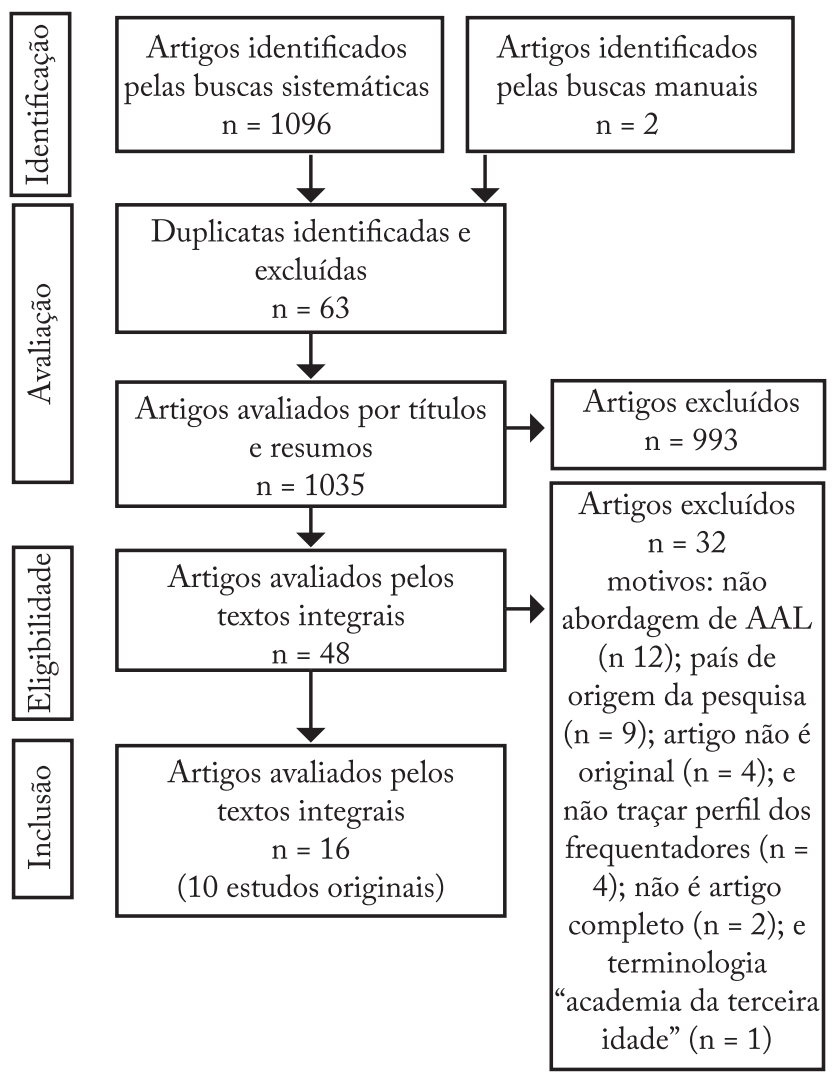

Figura 1 - Fluxograma da revisão de escopo 
avaliados pelos seus títulos e resumos, considerando o somatório dos registros recuperados pelas buscas nas bases de dados eletrônicas ( $\mathrm{n}=1.033$ ) e identificação manual $(n=2)$. Dos 48 artigos que remanesceram desta primeira etapa, 32 foram excluídos ao longo do processo de leitura dos seus textos integrais, tendo como principais motivos: não abordagem de $\mathrm{AAL}(\mathrm{n}=12)$ e país de origem $(n=9)$. Dessa forma, a síntese foi composta por 16 artigos originais ${ }^{13-28}$, todos de delineamento transversal, desenvolvidos no território brasileiro (Figura 1).

Entretanto, dentre os 16 artigos disponíveis, foram identificadas informações de 10 projetos originais, uma vez que, no processo de extração dos dados, os proje- tos conduzidos em Uruguaiana-Rio Grande do Sul ${ }^{13,14}$, Curitiba-Paraná ${ }^{19-22}$ e Pelotas-Rio Grande do Sul ${ }^{23,24}$ apresentaram mais de um artigo, com distintos objetivos e variáveis. Mesmo assim, para evitar a perda de informações relevantes, todos os artigos foram considerados para a presente síntese descritiva (Tabela 1).

Por localização, todas as regiões do país são representadas, envolvendo dados de cinco capitais ${ }^{15,18,19-22,25,26,28}$. Em relação aos objetivos, todos os projetos apresentaram artigos com objetivo de identificar o perfil dos frequentadores das AAL. O número de AAL analisadas variou entre $1^{17}$ e $58^{28}$, com amostras entre $30^{16}$ e $2.232^{19}$ pessoas. Os dados dos projetos foram coletados entre 2012 e 2018, com tempos de coleta entre

Tabela 1 - Informações descritivas dos projetos selecionados para a síntese, por ano de publicação.

\begin{tabular}{|c|c|c|c|c|c|c|c|c|}
\hline $\begin{array}{l}\text { Autor/ } \\
\text { projeto }^{\text {ref }}\end{array}$ & Ano & Cidade & Objetivos & Coleta & Meses & Estação & $\mathrm{AAL} *$ & $\mathrm{n}$ \\
\hline $\begin{array}{l}\text { Bergmann et } \\
\text { al. }{ }^{13,14}\end{array}$ & 2020 & $\begin{array}{l}\text { Uruguaiana-Rio Grande } \\
\text { do Sul }\end{array}$ & $\begin{array}{l}\text { Examinar a prevalência e correlatos } \\
\text { demográficos, socioeconômicos e de saúde } \\
\text { de frequentadores das } \mathrm{AAL}^{13} \text {; Identificar } \\
\text { barreiras para a utilização das } \mathrm{AAL}^{14}\end{array}$ & 2016 & $\begin{array}{l}\text { Setembro a } \\
\text { outubro }\end{array}$ & Primavera & 4 & 431 \\
\hline $\begin{array}{l}\text { Silva e } \\
\text { Anjos } 28\end{array}$ & 2020 & $\begin{array}{l}\text { Campo Grande-Mato } \\
\text { Grosso do Sul }\end{array}$ & $\begin{array}{l}\text { Identificar o perfil dos frequentadores, } \\
\text { investigar os espaços utilizados, avaliar } \\
\text { os motivos para a utilização e identificar } \\
\text { barreiras para a utilização das AAL }\end{array}$ & 2018 & $\begin{array}{l}\text { Fevereiro a } \\
\text { abril }\end{array}$ & Verão & 58 & 275 \\
\hline $\begin{array}{l}\text { Guzzo } \\
\text { Júnior e } \\
\text { Silva }^{16}\end{array}$ & 2019 & Castanhal-Pará & $\begin{array}{l}\text { Compreender o perfil dos frequentadores e } \\
\text { o discurso }\end{array}$ & 2017 & $\begin{array}{l}\text { Setembro a } \\
\text { outubro }\end{array}$ & Primavera & 2 & 30 \\
\hline $\begin{array}{l}\text { Mathias et } \\
\text { al. }{ }^{17}\end{array}$ & 2019 & Paranaguá-Paraná & $\begin{array}{l}\text { Identificar o perfil dos frequentadores e os } \\
\text { motivos para a prática de AF }\end{array}$ & 2014 & $\begin{array}{l}\text { Maio a } \\
\text { dezembro }\end{array}$ & $\begin{array}{l}\text { Inverno, } \\
\text { primavera e } \\
\text { verão }\end{array}$ & 1 & 64 \\
\hline $\begin{array}{l}\text { Silva, Tribess } \\
\text { e Papini }{ }^{27}\end{array}$ & 2019 & Uberaba-Minas Gerais & $\begin{array}{l}\text { Identificar o perfil dos frequentadores, nível } \\
\text { de AF e padrão de utilização }\end{array}$ & 2017 & $\begin{array}{l}\text { Setembro a } \\
\text { novembro }\end{array}$ & Primavera & 9 & 148 \\
\hline $\begin{array}{l}\text { Pinheiro } \\
\text { e Coelho } \\
\text { Filho }^{18}\end{array}$ & 2017 & Fortaleza-Ceará & $\begin{array}{l}\text { Identificar o perfil dos frequentadores das } \\
\text { AAL }^{* *}\end{array}$ & $\begin{array}{l}2015- \\
2016\end{array}$ & $\begin{array}{l}\text { Outubro a } \\
\text { abril }\end{array}$ & $\begin{array}{l}\text { Primavera, } \\
\text { verão e } \\
\text { outono }\end{array}$ & 11 & 374 \\
\hline $\begin{array}{l}\text { Projeto de } \\
\text { Teresina }{ }^{25,26}\end{array}$ & $\begin{array}{l}2017- \\
2020\end{array}$ & Teresina-Piauí & $\begin{array}{l}\text { Identificar o perfil dos frequentadores e } \\
\text { o padrão de utilização }{ }^{25} \text {; Caracterizar o } \\
\text { processo de implementação, utilização e } \\
\text { referenciamento da população às AAL, pela } \\
\text { Atenção Básica à Saúde }{ }^{26}\end{array}$ & 2015 & $\begin{array}{l}\text { Abril a } \\
\text { outubro }\end{array}$ & $\begin{array}{l}\text { Outono, } \\
\text { inverno e } \\
\text { primavera }\end{array}$ & 22 & 308 \\
\hline $\begin{array}{l}\text { Costa, } \\
\text { Freitas e } \\
\text { Silva }^{15}\end{array}$ & 2016 & $\begin{array}{l}\text { Florianópolis-Santa } \\
\text { Catarina }\end{array}$ & $\begin{array}{l}\text { Identificar o perfil dos frequentadores, } \\
\text { percepção de uso dos equipamentos e prática } \\
\text { de AFMV }\end{array}$ & 2013 & $\begin{array}{l}\text { Fevereiro a } \\
\text { abril }\end{array}$ & $\begin{array}{l}\text { Verão e } \\
\text { outono }\end{array}$ & 2 & 217 \\
\hline $\begin{array}{l}\text { Projeto de } \\
\text { Pelotas }^{23,24}\end{array}$ & $\begin{array}{l}2015- \\
2017\end{array}$ & $\begin{array}{l}\text { Pelotas-Rio Grande do } \\
\text { Sul }\end{array}$ & $\begin{array}{l}\text { Identificar o perfil dos frequentadores } \\
\text { Verificar a prática de } \mathrm{AF} \text { e seus fatores } \\
\text { associados }^{24}\end{array}$ & 2013 & $\begin{array}{l}\text { Outubro a } \\
\text { dezembro }\end{array}$ & Primavera & 3 & 323 \\
\hline $\begin{array}{l}\text { Projeto de } \\
\text { Curitiba }^{19-22}\end{array}$ & $\begin{array}{l}2014- \\
2019\end{array}$ & Curitiba-Paraná & $\begin{array}{l}\text { Identificar o perfil dos frequentadores } \\
\text { e o padrão de uso }{ }^{19} \text {; Verificar os fatores } \\
\text { associados à ocorrência de lesões durante } \\
\text { a prática de } \mathrm{AF}^{20} \text {; Analisar as possíveis } \\
\text { associações entre a distância da residência } \\
\text { até as } \mathrm{AAL} \text { com o uso destas estruturas } \\
\text { e a prática de } \mathrm{AF}^{21} \text { e Verificar padrões de } \\
\text { utilização e níveis de } \mathrm{AF}^{22}\end{array}$ & 2012 & $\begin{array}{l}\text { Outubro a } \\
\text { dezembro }\end{array}$ & Primavera & 20 & $\begin{array}{l}328- \\
2.232\end{array}$ \\
\hline
\end{tabular}

*número de $\mathrm{AAL} ;{ }^{* *}$ com frequência mínima de um mês nas AAL; AAL = academias ao ar livre; $\mathrm{AF}$ = atividade física; $\mathrm{AFMV}=$ atividade física de intensidade moderada à vigorosa; $\mathrm{n}=$ número de participantes 
dois $^{13,14,16}$ e oito ${ }^{17}$ meses (Tabela 1 ).

Foi observada grande heterogeneidade entre os procedimentos adotados nas coletas de dados, em relação à estação climática do ano, número de dias e os dias da semana que as coletas de dados ocorreram, tal como em relação aos horários escolhidos para a coleta. Não diferente, também se observou heterogeneidade entre os instrumentos utilizados para identificar o perfil da amostra e mensuração da AF, destacando-se a utilização de questionários próprios em todos os projetos (Tabela 2).

Quanto ao perfil dos frequentadores das AAL (Tabela 3), se indicou a maioria de mulheres entre as $\operatorname{amostras}^{13,15,16,18,22,23,25,27,28}$, pessoas na faixa etária entre
40 e 59 anos de idade ${ }^{13,16,22,25,27,28}$, com ensino médio completo $^{13,18,23,25,27}$ e casados ${ }^{18,22,23,27,28}$. Por outro lado, identificou-se grande heterogeneidade entre as rendas. Mesmo que a autopercepção de saúde seja boa ${ }^{22,23,27}$, os dados de IMC apontam, mais frequentemente, para o sobrepeso ${ }^{16,17,22,23,25}$, assim como às prevalências de hipertensão arterial (entre $27,7 \%{ }^{25}$ e $45,8 \%{ }^{23}$ ) e diabetes tipo II (entre $10,5 \%{ }^{23}$ e $16,2 \%{ }^{27}$ ).

$\mathrm{Na}$ Tabela 4, observa-se que os respondentes utilizam as AAL, por, pelo menos, 12 meses $^{18,22,23,26,28}$, com maior frequência de utilização nos dias úteis da semana ${ }^{25,27}$ e periodicidade de, pelo menos, três dias da semana ${ }^{15-18,22,23,25,27}$, com elevada heterogeneidade em relação ao tempo de utilização por sessão. Eles se lo-

Tabela 2 - Procedimentos e instrumentos utilizados para a coleta de dados dos projetos selecionados para a síntese.

\begin{tabular}{|c|c|c|c|}
\hline Autor/projeto ${ }^{\text {ref }}$ & Entrevistas & Procedimentos de coleta & Instrumentos de avaliação \\
\hline Bergmann et al. ${ }^{13,14}$ & Residências & Coleta com moradores do entorno de $500 \mathrm{~m}$ das AAL & $\begin{array}{l}\text { Questionário com } 3 \text { blocos*: } \\
\text { Características dos participantes } \\
\text { Utilização das AAL } \\
\text { AF - módulo de lazer do IPAQ }\end{array}$ \\
\hline Silva e Anjos ${ }^{28}$ & In loco & Coleta pela manhã, tarde e noite & $\begin{array}{l}\text { Questionário com perguntas sobre: } \\
\text { Forma de utilização } \\
\text { Impacto das AAL }\end{array}$ \\
\hline Guzzo Júnior e Silva ${ }^{16}$ & In loco & $\begin{array}{l}\text { Coleta nos dois horários de maior frequência }(6-7 \text { h30 } \\
\text { e } 17 \text { h30-19h) }\end{array}$ & $\begin{array}{l}\text { Questionário com respostas abertas em } 2 \text { blocos*: } \\
\text { Dados gerais } \\
\text { Identificação do discurso (motivações, benefícios e } \\
\text { sensações) }\end{array}$ \\
\hline Mathias et al. ${ }^{17}$ & In loco & Coleta aos sábados, em um horário (17-18h30) & $\begin{array}{l}\text { Questionário sobre*: } \\
\text { Perfil antropométrico, de saúde/doença e condição social; } \\
\text { Perfil de atividade humana (nível de AF) } \\
\text { Inventário sobre motivação para a AF }\end{array}$ \\
\hline Silva, Tribess e Papini ${ }^{27}$ & In loco & $\begin{array}{l}\text { Coleta em três dias (dois } \\
\text { dias de semana e um de final de semana, em três } \\
\text { horários }(7-9 \mathrm{~h}, 11-12 \mathrm{~h} \text { e } 17-19 \mathrm{~h})\end{array}$ & $\begin{array}{l}\text { Questionário divido em } 3 \text { domínios: } \\
\text { Caracterização dos participantes } \\
\text { Nível de AF e utilização das AAL } \\
\text { Percepção do ambiente }\end{array}$ \\
\hline $\begin{array}{l}\text { Pinheiro e Coelho } \\
\text { Filho }^{18}\end{array}$ & In loco & $\begin{array}{l}\text { Coletas em dias de semana e em dois horários (5-10h } \\
\text { e 16-21h) }\end{array}$ & Questionário* \\
\hline Projeto de Teresina $a^{25,26}$ & In loco & $\begin{array}{l}\text { Coleta em quatro visitas semanais em dias da semana } \\
\text { (início da manhã e final da tarde), obedecendo a } \\
\text { dinâmica de cada território }\end{array}$ & $\begin{array}{l}\text { Questionário formulado com base no estudo de Iepsen } \\
\text { e Silva }{ }^{23}\end{array}$ \\
\hline Costa, Freitas e Silva ${ }^{15}$ & In loco & $\begin{array}{l}\text { Em uma AAL a coleta ocorreu em cinco dias. } \\
\text { Na outra, em } 30 \text { dias, em três horários (7-12h; 14-17h } \\
\text { e } 18-21 \mathrm{~h})\end{array}$ & $\begin{array}{l}\text { Questionário com } 3 \text { blocos*: } \\
\text { Características dos participantes } \\
\text { Prática de AF habitual } \\
\text { Utilização das AAL }\end{array}$ \\
\hline Projeto de Pelotas ${ }^{23,24}$ & In loco & $\begin{array}{l}\text { Coletas em três dias (dois dias da semana e um do } \\
\text { final de semana, em dois horários } 8-11 \text { h e } 17-19 \text { h) }\end{array}$ & $\begin{array}{l}\text { Questionário formulado com base nos estudos de Souza } \\
\text { et al. } .^{22} \text {; Chrestani et al.** e IPAQ }\end{array}$ \\
\hline Projeto de Curitiba ${ }^{19-22}$ & In loco & $\begin{array}{l}\text { Coletas em quatro dias (dois dias da semana e dois do } \\
\text { final de semana, em quatro horários: 8-9h; 11-12h; } \\
14-15 \text { h e } 17-18 \mathrm{~h} \text { ) }\end{array}$ & $\begin{array}{l}\text { Questionário onde foram mensurados diversos } \\
\text { indicadores*: } \\
\text { AF - módulo de lazer do IPAQ } \\
\text { Qualidade de vida } \\
\text { Padrão e orientação para a utilização } \\
\text { Informações pessoais e sociodemográficas }^{19} \\
\text { Esforço percebido } \\
\text { SOPARC }\end{array}$ \\
\hline
\end{tabular}

*questionário desenvolvido para o projeto; **Chrestani, Santos, Matijasevich44; AAL = Academias ao Ar Livre; AF = atividade física; IPAQ= International Physical Activity Questionnaire; SOPARC = System for Observing of Play and Recreation in Communities. 
comovem ativamente (ex. caminhada, bicicleta) para as AAL $^{17,21,27}$ e têm autopercepção de segurança pública para a utilização da $\mathrm{AAL}^{18,22,27}$. No projeto conduzido em Uruguaiana-Rio Grande do Sul, mais especificamente, observou-se que os períodos matutino e vespertino oferecem melhores percepções de segurança pública, quando comparados ao noturno ${ }^{22}$. Em relação à $\mathrm{AF}$, observou-se a prática de caminhada ${ }^{18,22,25}$ assim como a realização destas atividades no tempo de lazer $^{13,23,27}$.

\section{Discussão}

A partir dos dados de 10 projetos originais, que representam localidades nas cinco distintas regiões do país, a presente síntese apontou que mulheres, pessoas na faixa etária entre 40 e 59 anos de idade, com ensino médio completo, casadas e com sobrepeso representam o perfil mais observado do público que utiliza as AAL no país. Complementarmente, a síntese também aponta que os

Tabela 3 - Informações socioeconômicas e de saúde dos frequentadores das academias ao ar livre

\begin{tabular}{|c|c|}
\hline $\begin{array}{l}\text { Variáveis (número de projetos } \\
\text { com dados disponíveis) }\end{array}$ & Resultados \\
\hline $\operatorname{Sexo}(n=10)$ & $\begin{array}{l}\text { Mulheres }(\mathrm{n}=9)^{13,15,16,18,22,23,25,27,28} \\
\text { Homens }(\mathrm{n}=1)^{17}\end{array}$ \\
\hline Faixa etária, em anos $(\mathrm{n}=10)$ & $\begin{array}{l}\text { Média: } 53,8(\mathrm{n}=1)^{25} \\
\text { Frequências: } 18-39(\mathrm{n}=1)^{17} ; 40-49(\mathrm{n}= \\
2)^{13,16} ; 40-59(\mathrm{n}=3)^{22,27,28} ; \geq 50(\mathrm{n}=1)^{23} ; \\
50-59(\mathrm{n}=1)^{15} ; 60-69^{18}\end{array}$ \\
\hline Cor da pele $(\mathrm{n}=3)$ & Branca $(\mathrm{n}=2)^{18,23} ;$ Parda $(\mathrm{n}=1)^{25}$ \\
\hline Escolaridade $(n=7)$ & $\begin{array}{l}\text { Ensino Médio Completo }(\mathrm{n}= \\
5)^{13,18,23,25,27} \text { Ensino Superior Completo } \\
(\mathrm{n}=2)^{15,21}\end{array}$ \\
\hline Estado civil $(\mathrm{n}=6)$ & $\begin{array}{l}\text { Casados }(\mathrm{n}=5)^{18,22,23,27,28} \text {; Solteiros ( } \mathrm{n} \\
=1)^{17}\end{array}$ \\
\hline Renda mensal $(n=5)$ & $\begin{array}{l}\text { Individual: Até } 1 \text { salário mínimo }(\mathrm{n}= \\
1)^{16} ; \text { Entre } \mathrm{R} \$ 1.319,00 \text { e } 2.256,00(\mathrm{n} \\
=1)^{23} ; \text { Entre acima de } 2 \text { a } 5 \text { salários } \\
\text { mínimos }(\mathrm{n}=1)^{27} \\
\text { Familiar }(\mathrm{n}=2) \text { : Até } 2 \text { salários mínimos } \\
(\mathrm{n}=1)^{13} ; \text { Dados variados no projeto de } \\
\text { Curitiba (entre } \mathrm{R} \$ 1.000,00 \text { e } 2.999,00^{22} ; \\
\left.\leq 2.999,00^{20} \text { e } \geq 3.000,00^{21}\right)\end{array}$ \\
\hline Percepção de saúde $(n=4)$ & Excelente $(\mathrm{n}=1)^{13 *}, \mathrm{Boa}(\mathrm{n}=3)^{22,23,27}$ \\
\hline $\begin{array}{l}\text { Índice de Massa Corporal } \\
(\mathrm{n}=7)\end{array}$ & $\begin{array}{l}\text { Médias: } 25,9(\mathrm{n}=1)^{25} ; 26(\mathrm{n}=1)^{17} \\
\text { Classificações: Eutróficos }(\mathrm{n}=1)^{18} ; \\
\text { Excesso de peso }(\mathrm{n}=1)^{22} ; \text { Sobrepeso }(\mathrm{n}= \\
2)^{16,23} ; \text { Obesos }(\mathrm{n}=1)^{13}\end{array}$ \\
\hline Hipertensão $(\mathrm{n}=4)$ & Prevalência: $27,7^{25} ; 33,8^{27} ; 40,9^{18} ; 45,8^{23}$ \\
\hline Diabetes tipo II $(n=4)$ & Prevalência: $10,5^{23} ; 11,5^{18} ; 12,4^{25} ; 16,2^{27}$ \\
\hline Hábito tabagista $(\mathrm{n}=3)$ & Não fumantes $(\mathrm{n}=3)^{17,18,23}$ \\
\hline
\end{tabular}

*pessoas que utilizam as academias ao ar livre por, pelo menos, duas vezes na semana. respondentes reportaram a utilização das AAL há, pelo menos, 12 meses, com periodicidade de uso de, pelo menos, três dias da semana, geralmente, nos dias úteis.

Em relação aos métodos, observou-se grande heterogeneidade no número de AAL abordadas e nos procedimentos de coleta e instrumentos utilizados para avaliação da AF. Por outro lado, as informações disponíveis não são conclusivas para: renda, motivos para utilização das AAL e, companhia/suporte social para sua utilização. Complementarmente, em vista do caráter quantitativo dos projetos, sugere-se que projetos futuros podem avançar na identificação dos frequentadores por meio da utilização de abordagens mistas (qualitativas e quantitativas) nas coletas e análises dos dados, no sentido de aprofundar também questões sobre motivações, gostos, preferências, limitações e modos de utilização.

$\mathrm{O}$ fato de a coleta de dados mais antiga ter sido realizada no ano de 2012 pode ser justificada, uma vez que a disseminação das AAL se enquadra como uma

Tabela 4 - Informações sobre o uso das academias ao ar livre e prática de atividade física.

\begin{tabular}{|c|c|}
\hline $\begin{array}{l}\text { Variáveis (número de projetos } \\
\text { com dados disponíveis) }\end{array}$ & Resultados \\
\hline $\begin{array}{l}\text { Tempo que frequenta a } \mathrm{AAL} \\
(\mathrm{n}=6)\end{array}$ & $\begin{array}{l}\text { Média: } 22,2 \text { meses }(n=1)^{26} \\
\text { Frequências: Entre } 1 \text { e } 12 \text { meses }(n=1)^{27} \text {; } \\
>12 \text { meses }(n=3)^{18,22,23} ; \text { Entre } 12 \text { e } 36 \\
\text { meses }(n=1)^{28}\end{array}$ \\
\hline $\begin{array}{l}\text { Periodicidade, em dias da } \\
\text { semana, nas AAL }(n=8)\end{array}$ & $\begin{array}{l}2-3(\mathrm{n}=1)^{28} ; \geq 3(\mathrm{n}=3)^{17,22,23,27} ; 3-5(\mathrm{n}= \\
1)^{25} ; \geq 5(\mathrm{n}=1)^{16,18} ; 5-7(\mathrm{n}=1)^{15}\end{array}$ \\
\hline \multirow{2}{*}{$\begin{array}{l}\text { Tempo de permanência por } \\
\text { sessão nas AAL }(n=6)\end{array}$} & Média: $33 \mathrm{~min} / \mathrm{d}(\mathrm{n}=1)^{23}$; \\
\hline & $\begin{array}{l}\text { Frequências: } \geq 30 \mathrm{~min} / \mathrm{d}(\mathrm{n}=1)^{27} ; \leq 30 \\
\mathrm{~min} / \mathrm{d}(\mathrm{n}=2)^{18,22} ; 20-40 \mathrm{~min} / \mathrm{d}(\mathrm{n}=1)^{25} \\
40-60 \mathrm{~min} / \mathrm{d}(\mathrm{n}=1)^{28} ; 150 \mathrm{~min} / \mathrm{sem}(\mathrm{n}= \\
1)^{15} ; \geq 60 \mathrm{~min} / \mathrm{d}(\mathrm{n}=1)^{17}\end{array}$ \\
\hline $\begin{array}{l}\text { Períodos da semana / Dias de } \\
\text { utilização }(n=3)\end{array}$ & $\begin{array}{l}\text { Dias da semana }(n=2)^{25,27} ; \text { Dias da } \\
\text { semana e do final de semana }(n=1)^{22}\end{array}$ \\
\hline $\begin{array}{l}\text { Meio de locomoção para as } \\
\text { AAL }(n=3)\end{array}$ & Ativo $(\mathrm{n}=3)^{17,21,27}$ \\
\hline $\begin{array}{l}\text { Companhia para as atividades } \\
\text { na AAL }(\mathrm{n}=3)\end{array}$ & $\operatorname{Sim}(\mathrm{n}=1)^{18} ;$ Não $(\mathrm{n}=2)^{20,27}$ \\
\hline $\begin{array}{l}\text { Principais motivos para } \\
\text { frequentar as AAL }(n=3)\end{array}$ & $\begin{array}{l}\text { Manutenção da saúde, recomendação } \\
\text { médica e redução do peso }(n=1)^{25} \text {; Prazer, } \\
\text { Saúde e Estética }(n=1)^{17} \text {, Ser um espaço } \\
\text { ao ar livre e não uma sala fechada com } \\
\text { equipamentos e Gratuidade }(n=1)^{16}\end{array}$ \\
\hline $\begin{array}{l}\text { Percepção de segurança } \\
\text { pública }(\mathrm{n}=3)\end{array}$ & $\operatorname{Sim}(n=3)^{18,22^{*}, 27}$ \\
\hline Prática de caminhada $(n=4)$ & $\begin{array}{l}0 \text { dias/semana }(n=1)^{23} ; \geq 150 \text { minutos/ } \\
\text { semana }(n=1)^{22} ; \operatorname{Sim}(n=2)^{18,25}\end{array}$ \\
\hline $\begin{array}{l}\text { Nível de AF no tempo de } \\
\text { lazer }\end{array}$ & Suficientemente ativo $(\mathrm{n}=3)^{13,23,27}$ \\
\hline
\end{tabular}

*Percepção de segurança boa nos turnos da manhã e tarde, sem percepção de segurança à noite; $\mathrm{AAL}=\mathrm{Academia}$ ao Ar Livre; $\mathrm{min} / \mathrm{d}$ = minutos diários. 
estratégia mais atual de acesso à AF no país. Complementarmente, o fato de ser uma estratégia atual também justifica, em alguns casos, o número reduzido de AAL em alguns dos projetos incluídos ${ }^{15-17,23,24}$.

Observando-se a maior frequência de mulheres e pessoas acima de 40 anos, as AAL podem ser entendidas como uma estratégia potencial para o acesso à $\mathrm{AF}$ neste grupo, visto que o corpo de evidências sugere a gradual redução da prática de AF com a idade, sobretudo entre as mulheres ${ }^{29}$. Por outro lado, mesmo que as tendências temporais sugiram o aumento dos níveis de AF suficiente no tempo de lazer em mulheres nos últimos anos ${ }^{8}$, ainda são percebidas diferenças em relação aos níveis de $\mathrm{AF}$ verificados em homens ${ }^{8,10}$. Mesmo que as AAL não necessariamente se configurem como uma estratégia específica aos grupos mais vulneráveis, a identificação do seu público frequentador pode apoiar futuras ações concernentes às AAL como uma estratégia para redução das desigualdades sociais e de gênero ${ }^{30}$ em relação à prática de $\mathrm{AF}$ no país.

Sobre a faixa etária mais prevalente entre as pesquisas disponíveis (40-59 anos de idade), que dialoga com trabalho de revisão recente ${ }^{4}$, foi confirmada uma das hipóteses da presente revisão, que as AAL não se configuram como um espaço específico para idosos ( $\geq$ 60 anos), assim como se sugere a antiga terminologia “academia da terceira idade". Dessa forma, um melhor acesso às AAL pode contribuir ao papel positivo à saúde desempenhado pela AF ao longo do envelhecimento, com particular enfoque nas associações positivas entre sua prática e à atenuação do declínio cognitivo, doenças neurodegenerativas ${ }^{31}$, assim como da fragilida$\mathrm{de}^{32} \mathrm{e}$ incapacidade funcional ${ }^{33}$. Complementarmente, em vista do gradual aumento da proporção de idosos na população total brasileira ${ }^{34}$, na expectativa de vida e na alteração do perfil de morbidade ${ }^{35}$, o incentivo à construção e/ou manutenção permanente das estruturas existentes das AAL pode representar um grande avanço dentro do conjunto de ações e estratégias já existentes de promoção da saúde nesta faixa etária.

Em relação ao padrão de utilização das AAL, a presente síntese corrobora a evidência de uma revisão prévia, constituída por evidências internacionais, que destaca a frequência entre três e cinco dias por semana ${ }^{36}$. Complementarmente, esta mesma revisão ${ }^{36}$ também traz outras evidências de interesse, como, em relação à distância das AAL das residências dos seus frequentadores (500-2.007 metros), que, de alguma forma pode estar relacionada à opção (e possibilidade) pelo trans- porte ativo para sua utilização ${ }^{17,21,27}$ e o risco de lesões, pela utilização inadequada das estruturas e/ou falta de orientação. Mesmo que alguns dos projetos tenham abordado estas temáticas ${ }^{13,14,20} \mathrm{e}$ até mesmo a investigação dos motivos de não-utilização entre as pessoas que moram próximas as $\mathrm{AAL}^{13,14}$, não se atingiu o número estipulado para sua apresentação na síntese.

Os dados da síntese também corroboram as evidências prévias que destacam, em adultos e idosos, associações entre maiores níveis de $\mathrm{AF}^{10,37}$ e escolaridade. Esforços podem ser investidos para que pessoas com menor escolaridade tenham maior acesso às AAL, uma vez que estas estruturas, geralmente, oferecem uma linguagem instrutiva simples, por meio de painéis informativos permanentes e ilustrativos que detalham, por exemplo, a forma correta de manusear os aparelhos para realização dos exercícios, os grupamentos musculares trabalhados e sugestões para sequências de exercícios. Um outro ponto, identificado no projeto de Teresina-Piauí ${ }^{25,26}$ e que pode ser investigado em projetos futuros, é sobre a importância da presença de um profissional de Educação Física nas AAL, que se reverteria em uma orientação especializada à utilização das estruturas.

Deve-se destacar também a inconclusividade dos dados relacionados à companhia/suporte social na utilização das AAL, abrindo margem para um aprofundamento desta questão nos futuros projetos. Revisão prévia destaca que as pessoas geralmente procuram as AAL com fins na promoção de saúde ${ }^{4}$, o que se confirmou nesta pesquisa, independente das motivações específicas. Entretanto, cabe pontuar que, em Souza \& Vendrusco$1 \mathrm{o}^{38}$, a manutenção dos idosos em um programa de AF dar-se-á para muito além da percepção dos benefícios à saúde, envolvendo também a percepção de ganhos mais ampliados, como "desenvolvimento pessoal, socialização e troca de informações, experiências, suporte e afeto”.

Alguns achados conflitantes sobre a saúde dos participantes das AAL também merecem atenção, destacando que, aparte da boa percepção de saúde ${ }^{22,23,27}$, observou-se maior frequência de pessoas com IMC na faixa do sobrepeso ${ }^{16,17,22,23,25}$, assim como as elevadas proporções de hipertensos e diabéticos. Nesse sentido, são necessárias estratégias que visam ampliação do uso das AAL, visto que podem se reverter em melhoras nos indicadores de massa corporal, reconhecendo-se a maior chance de morbimortalidade associadas ao sobrepeso e obesidade com o envelhecimento ${ }^{40,41}$, assim como da redução do consumo de medicamentos, uma vez que pessoas mais ativas tendem a consumir menos 
medicamentos quando comparadas às pessoas que não praticam $\mathrm{AF}^{42}$.

Ao se considerar que a utilização das AAL se dá de acordo com a dinâmica de cada território, desde os momentos iniciais da extração de dados percebeu-se que a heterogeneidade nos procedimentos (ex. dias da semana e/ ou final de semana; número de dias em que as coletas ocorreram por AAL e horários de coleta) seria uma importante condição de viabilidade para os projetos, assim como da necessidade da utilização de questionários próprios para obtenção de informações na especificidade das AAL. Por outro lado, também cabe destacar a lógica de substituição dos dias de coleta de acordo com as condições climáticas, uma vez que AAL geralmente se situam em espaços públicos abertos, sem a presença de cobertura, o que limita a permanência de frequentadores em dias muito quentes, muito frios e/ou chuvosos.

A presente revisão contém algumas limitações, a saber: a exclusão de artigos que partiram da terminologia "academia da terceira idade", uma vez que, em nossa avaliação, esta própria terminologia direcionaria, pelo menos no que tange à faixa etária, o resultado final. Revisão de estudos internacionais também aponta para distintas terminologias ${ }^{43}$, o que limita a elaboração de sínteses mais ampliadas. Por outro lado, este é um dos primeiros trabalhos de revisão com foco nas AAL em sua atual terminologia, de forma que a evidência apresentada sobre os diferentes indicadores de caracterização dos frequentadores possa orientar um debate mais específico sobre os estudos que as abordaram de tal maneira. A não realização da avaliação do risco de viés dos estudos originais também pode ser entendida como uma limitação. Entretanto, essa opção foi assumida em vista do caráter puramente descritivo deste trabalho, como mencionado nas seções anteriores.

Por fim, as evidências disponíveis permitem concluir que as AAL contribuem para a equidade ao acesso à AF. Entretanto, novos estudos são necessários são necessários, com utilização de abordagens mistas, para a elucidação de características que ao momento ainda são inconclusivas.

\section{Conflito de interesse}

Os autores declaram não haver conflito de interesse.

\section{Contribuição dos autores}

Battistel JA, Floss MI e Guerra PH, conceberam a ideia central do estudo; participaram da elaboração das buscas sistemáticas, avaliação dos títulos, resumos e textos integrais; do desenvolvimento da síntese, análise dos resultados e escrita do texto. Cruvinel AFP e Barbato PR participaram da revisão do texto. Fermino RC participou do desenvolvimento da síntese, análise dos resultados e revisão do texto.

\section{Referências}

1. Chandrabose M, Rachele JN, Gunn L, Kavanagh A, Owen $\mathrm{N}$, Turrell G, et al. Built environment and cardio-metabolic health: systematic review and meta-analysis of longitudinal studies. Obes Rev. 2019;20(1):41-54.

2. Umstattd Meyer MR, Bridges CN, Schmid TL, Hecht AA, Pollack Porter KM. Systematic review of how Play Streets impact opportunities for active play, physical activity, neighborhoods, and communities. BMC Public Health. 2019;19(1):335.

3. Van Hecke L, Ghekiere A, Veitch J, Van Dyck D, Van Cauwenberg J, Clarys P, et al. Public open space characteristics influencing adolescents' use and physical activity: A systematic literature review of qualitative and quantitative studies. Health Place. 2018;51:158-73.

4. Lee JLC, Lo TLT, Ho RTH. Understanding outdoor gyms in public open spaces: A systematic review and integrative synthesis of qualitative and quantitative evidence. Int J Environ Res Public Health. 2018;15(4).pii:E590.

5. Programa das Nações Unidas para o Desenvolvimento. Relatório de Desenvolvimento Humano Nacional sobre Atividades Físicas e Esportivas e Desenvolvimento Humano. 2016. Disponível em: < https://www.br.undp.org/content/ dam/brazil/docs/IDH/und-br-nota-conceitual-final-2016. pdf >. Acesso em 20 set. 2019.

6. Cruz MS, Bernal RTI, Claro RM. Tendência da prática de atividade física no lazer entre adultos no Brasil (2006-2016). Cad. Saúde Pública. 2018;34(10):e00114817.

7. Malta D, Andrade S, Santos M, Rodrigues G, Mielke G. Tendências dos indicadores de atividade física em adultos: Conjunto de capitais do Brasil 2006-2013. Rev Bras Ativ Fis Saude. 2015;20(2):141-51.

8. Florindo AA, Barbosa JPAS, Barrozo LV, Andrade DR, Aguiar BS, Failla MA, et al. Walking for transportation and built environment in Sao Paulo city, Brazil. J Transp Health. 2019;15:100611.

9. Casas RCRL, Bernal RTI, Jorge AO, Melo EM, Malta DC. Fatores associados à prática de atividade física na população brasileira - Vigitel 2013. Saude Debate. 2018;42(spe4):13444.

10. Silva TD, Souza SS, Starepravo FA. Academia da Saúde, Academia da Cidade e Academia ao Ar Livre nas agendas política e governamental dos estados brasileiros. Rev Bras Ativ Fís Saúde. 2020;25:e0163.

11. Barbosa Filho VC, Tricco AC. Scoping review: a relevant methodological approach for knowledge synthesis in Brazil's health literature. Rev Bras Ativ Fís Saúde. 2019;24:e0082.

12. Tricco AC, Lillie E, Zarin W, O’Brien KK, Colquhoun H, Levac D, et al. PRISMA Extension for Scoping Reviews (PRISMA-ScR): Checklist and Explanation. Ann Intern Med. 2018;169(7):467-473.

13. Bergmann GG, Streb AR, Ferrari M, Alves DCD, Soares BAC, Ferreira GD, et al. The use of outdoor gyms is associated with women and low-income people: a cross-sectional study. Public Health. 2021;190:16e22. 
14. Bergmann GG, Streb AR, Ferrari M, Alves DCD, Soares BAC, Ferreira GD, et al. Barriers for the use of outdoor gyms in adults and elderly from a southern city of Brazil. Rev Bras Ativ Fís Saúde. 2020;25:e0159.

15. Costa BGG, Freitas CR, Silva KS. Atividade física e uso de equipamentos entre usuários de duas Academias ao Ar Livre. Rev Bras Ativ Fis Saude 2016;21(1):29-38.

16. Guzzo Junior CCE, Silva WLA. Academias ao ar livre em Castanhal, uma opção de lazer e convívio social? O perfil e discurso do usuário. Licere. 2019;22:137-58.

17. Mathias NG, Melo Filho J, Skudlarek AC, Gallo LH, Fermino RC, Gomes AR. Motivos para a prática de atividades físicas em uma academia ao ar livre de ParanaguáPR. Rev Bras Cien Esporte. 2019;41(2):222-8.

18. Pinheiro WL, Coelho Filho JM. Perfil dos idosos usuários das academias ao ar livre para a terceira idade. Rev Bras Promoc Saude. 2017;30(1):93-101.

19. Alberico CO, Hipp JA, Reis RS. Association between neighborhood income, patterns of use, and physical activity levels in fitness zones of Curitiba, Brazil. J Phys Act Health. 2019;16(6):447-54.

20. Silva AT, Fermino RC, Alberico CO, Reis RS. Fatores associados à ocorrência de lesões durante a prática de atividade física em academias ao ar livre. Rev Bras Med Esporte. 2016;22(4):267-71.

21. Silva AT, Fermino RC, Lopes AAS, Alberico CO, Reis RS. Distance to fitness zone, use of facilities and physical activity in adults. Rev Bras Med Esporte. 2018;24(2):157-61.

22. Souza CA, Fermino RC, Añez CRR, Reis RS. Perfil dos frequentadores e padrão de uso das academias ao ar livre em bairros de baixa e alta renda de Curitiba-PR. Rev Bras Ativ Fis Saúde. 2014;19(1):86-97.

23. Iepsen AM, Silva MC. Perfil dos frequentadores das academias ao ar livre da cidade de Pelotas - RS. Rev. Bras Ativ Fis Saude. 2015;20(4):413-24.

24. Silva MC, Iepsen AM, Caputo EL, Engers PB, Spohr CF, Vilela GF, et al. Leisure-time physical activity and associated factors in fitness zones. Rev Bras Cineantropom Desempenho Hum. 2017;19(2):185-95.

25. Ibiapina ARL, Moura MN, Santiago MLE, Moura TNB. Caracterização dos usuários e do padrão de uso das academias ao ar livre. Rev Bras Promoc Saude. 2017;30(4):1-10.

26. Moura MN, Moura ARLI, Santiago MLE, Moura TNB. Academias ao ar livre: percepções dos usuários e relação com o serviço de saúde. Arq Cienc Saúde. 2020;24:87-94.

27. Silva DB, Tribess S, Papini CB. Perfil dos usuários e padrão de utilização das academias ao ar livre de Uberaba, Minas Gerais. Rev Bras Ativ Fís Saúde. 2019;24:e0111.

28. Silva JVP, Anjos VAA. The impacts of outdoor gyms on leisure physical activity in Campo Grande-MS. Holos. 2020;6:e10180.

29. Sun F, Norman IJ, While A. Physical activity in older people: a systematic review. BMC Public Health. 2013;13:449.

30. Programa das Nações Unidas para o Desenvolvimento. Movimento é vida: Atividades físicas e esportivas para todas as pessoas. Atividades físicas e esportivas e Mulheres no Brasil. 2017. Disponível em: < http://movimentoevida.org/wpcontent/uploads/2017/09/Atividades-Fi\%CC\%81sicas-eEsportivas-e-Ge\%CC\%82nero.pdf>. Acesso em: 16 set. 2019.
31. Bherer L, Erickson KI, Liu-Ambrose T. A review of the effects of physical activity and exercise on cognitive and brain functions in older adults. J Aging Res. 2013;2013:657508.

32. Costa TB, Neri AL. Medidas de atividade física e fragilidade em idosos: dados do FIBRA Campinas, São Paulo, Brasil. Cad. Saude Publica. 2011;27(8),1537-1550.

33. Virtuoso Júnior JS, Guerra RO. Incapacidade funcional em mulheres idosas de baixa renda. Rev. Ciência e Saúde Coletiva. 2011;16(5):2541-2548.

34. Marinho F, Passo VMA, França EB. Novo século, novos desafios: mudança no perfil da carga de doença no Brasil de 1990 a 2010. Epidemiol. Serv Saude. 2016;25(4),713-24.

35. Geib LTC. Determinantes sociais da saúde do idoso. Rev. Ciência e Saúde Coletiva. 2012;17(1),123-33.

36. Fernández-Rodríguez EF, Merino-Marban R, RomeroRamos O, López-Fernández I. A systematic review about the characteristics and patterns of use of outdoor gyms. J Hum Sport Exerc. 2020;15:S688-S707.

37. Peixoto SV, Mambrini JVM, Firmo JOA, Loyola Filho AI, Souza Junior PRB, Andrade FB, et al. Prática de atividade física entre adultos mais velhos: resultados do ELSI-Brasil. Rev Saude Publica. 2018;52(Suppl.2),5s.

38. Souza DL, Vendrusculo R. Fatores determinantes para a continuidade da participação de idosos em programas de atividade física: a experiência dos participantes do projeto "Sem Fronteiras”. Rev Bras Educ Fís Esporte. 2010;24(1):95-105.

39. Giehl MWC, Schneider IJC, Corseuil HX, Benedetti TRB, Orsi E. Atividade física e percepção do ambiente em idosos: estudo populacional em Florianópolis. Rev Saude Publica. 2012;46(3),516-25.

40. Donini LM, Savina C, Gennaro E, De Felice MR, Rosano A, Pandolfo MM, et al. A systematic review of the literature concerning the relationship between obesity and mortality in the elderly. J Nutr Health Aging. 2012;16(1):89-98.

41. Russo C, Jin Z, Homma S, Rundek T, Elkind MS, Sacco RL, et al. Effect of obesity and overweight on left ventricular diastolic function: a community-based study in an elderly cohort. J Am Coll Cardiol. 2011;57(12):1368-74.

42. Bertoldi AD, Barros AJD, Hallal PC, Lima RC. Utilização de medicamentos em adultos: prevalência e determinantes individuais. Rev. de Saúde Pública. 2004;38(2),228-38.

43. Jansson AK, Lubans DR, Smith JJ, Duncan MJ, Haslam R, Plotnikoff RC. A systematic review of outdoor gym use: Current evidence and future directions. J Sci Med Sport. 2019;22(12):1335-43.

44. Chrestani MAD, Santos IS, Matijasevich AM. Hipertensão arterial sistêmica auto-referida: validação diagnóstica em estudo de base populacional. Cad. Saúde Pública. 2009;25(11):2395-2406.

Recebido: $19 / 10 / 2020$

Aprovado: 15/02/2021 